\title{
ON APPROXIMATE GENERALIZED LIE DERIVATIONS
}

\author{
JANUSZ BRZDĘK AND AJdA FOŠNER \\ Pedagogical University, Poland and University of Primorska, Slovenia
}

\begin{abstract}
Motivated by the notion of the Hyers-Ulam stability, we prove results that are efficient tools for the study of approximate generalized Lie derivations on Lie algebras. We also provide simple examples of applications of our outcomes. In particular, we obtain some auxiliary results on the stability of the additive Cauchy equation.
\end{abstract}

\section{INTRODUCTION}

One of the fundamental questions in the theory of the stability of functional equations is: When is it true that a mapping, which satisfies a functional equation approximately, must be close to an exact solution of that equation? It is related to a problem raised by Ulam (cf. [13,26]) concerning the stability of group homomorphisms. This question was partially affirmatively answered by Hyers ([13]) in Banach spaces. Subsequently, the result of Hyers was generalized by Aoki ([2], see also [25]). For further information about the topic we refer the reader to, e.g., $[5,14,17]$.

The first result of this area is due to Jun and Park (see [18]) who investigated approximate derivations of $C^{n}([0,1])$. Further, Badora [3] studied the Hyers-Ulam stability of derivations acting between Banach algebras. During the past few years, approximate derivations were studied by a number of mathematicians (see $[1,3,4,10,11,20-22,24]$ and references therein). Following this line of investigations, we prove several results on functions that satisfy the conditions, defining the generalized Lie derivations, only approximately.

The paper is organized as follows. First we fix the notations and give basic definitions. In section 2 we obtain some auxiliary results on the stability of the additive Cauchy equation. In section 3 we prove our main results on the

2010 Mathematics Subject Classification. 16W20, 16W25, 39B62, $39 \mathrm{~B} 82$.

Key words and phrases. Stability, normed algebra, Banach bimodule, Lie derivation, generalized Lie derivation. 
stability of generalized Lie derivations and, at the end of the paper, in section 4 , we present three simple examples of applications of them.

Throughout the paper, $\mathcal{A}$ will represent a Lie algebra over the real or complex field $\mathbb{F}$ and $\mathcal{M}$ a Banach $\mathcal{A}$-bimodule. For $x \in \mathcal{A}$ and $y \in \mathcal{M}$, the symbol $[x, y]$ will denote the commutator $x y-y x \in \mathcal{M}$. We say that an additive mapping $d: \mathcal{A} \rightarrow \mathcal{M}$ is a Lie derivation if

$$
d([x, y])=[d(x), y]+[x, d(y)], \quad x, y \in \mathcal{A} .
$$

Hvala ([12]) has introduced the notion of generalized Lie derivations as follows. An additive mapping $g: \mathcal{A} \rightarrow \mathcal{M}$ is called a generalized Lie derivation if there exists an additive mapping $d: \mathcal{A} \rightarrow \mathcal{M}$ such that

$$
g([x, y])=g(x) y-g(y) x+x d(y)-y d(x), \quad x, y \in \mathcal{A} .
$$

For the sake of precision, given an additive $d: \mathcal{A} \rightarrow \mathcal{M}$, every additive $g: \mathcal{A} \rightarrow \mathcal{M}$ satisfying (1.1) will be named a generalized Lie $d$-derivation.

Of course, the class of generalized Lie derivations covers both the class of Lie derivations and the class of generalized derivations. The definition of generalized Lie derivations was suggested by Brešar (see [12]). Related yet somewhat different definitions of generalized Lie derivations were introduced also by Nakajima ([23]).

\section{Auxiliary Results}

Before stating our auxiliary theorems, let us introduce some basic definitions and known results, which we will use in the sequel.

Let $\mathfrak{B}_{\mathbb{F}}$ stand for the family of all sets $\Gamma \subseteq \mathbb{F}$ such that each additive function $f: \mathbb{F} \rightarrow \mathcal{M}$ that is bounded on $\Gamma$ must be continuous. It is wellknown that if $\Gamma \subset \mathbb{F}$ and int $\Gamma \neq \emptyset$, then $\Gamma \in \mathfrak{B}_{\mathbb{F}}$. This is also the case when $\Gamma \subseteq \mathbb{F}$ has a positive inner Lebesgue measure or contains a subset of the second category and with the Baire property (cf. [19]). For more information on $\mathfrak{B}_{\mathbb{F}}$ and further references concerning the subject we refer the reader to, e.g., $[15,16,19]$.

We say that an additive mapping $f: \mathcal{A} \rightarrow \mathcal{M}$ is $\mathbb{F}$-linear if $f(\lambda x)=\lambda f(x)$ for all $x \in \mathcal{A}$ and all scalars $\lambda \in \mathbb{F}$.

Lemma 2.1 ([8, Lemma 1$])$. Let $\mathbb{F}=\mathbb{C}, \Gamma_{0} \in \mathfrak{B}_{\mathbb{C}}$ be a bounded set and let $f: \mathcal{A} \rightarrow \mathcal{M}$ be an additive function such that $f(\lambda x)=\lambda f(x)$ for all $x \in \mathcal{A}$ and $\lambda \in \Gamma_{0}$. Then $f$ is $\mathbb{C}$-linear.

Let $\mathbb{S}:=\{\lambda \in \mathbb{C}:|\lambda|=1\}$. Using the results of $[15,16]$ one can also obtain the following.

Corollary 2.2. Let $\mathbb{F}=\mathbb{C}, \Gamma$ be a connected nontrivial subset of $\mathbb{S}$, and $f: \mathcal{A} \rightarrow \mathcal{M}$ be an additive function such that $f(\lambda x)=\lambda f(x)$ for all $x \in \mathcal{A}$ and all $\lambda \in \Gamma$. Then $f$ is $\mathbb{C}$-linear. 
In the proof of our first theorem we will use a fixed point result from [7]. To simplify its presentation we need the following two hypotheses. Here, $X$ is a nonempty subset of $\mathcal{A}, p, q: X \rightarrow X$, and $\lambda, \nu \in \mathbb{F}$. As usual, $A^{B}$ denotes the family of all functions mapping a set $B \neq \emptyset$ into a set $A \neq \emptyset$ and $2^{D}$ stands for the family of all subsets of a set $D$.

(H1) $\mathcal{T}: \mathcal{M}^{X} \rightarrow \mathcal{M}^{X}$ is an operator satisfying

$$
\begin{aligned}
\|(\mathcal{T} \zeta)(x)-(\mathcal{T} \eta)(x)\| \leq & |\lambda|\|\zeta(p(x))-\eta(p(x))\| \\
& +|\nu|\|\zeta(q(x))-\eta(q(x))\|, \quad \zeta, \eta \in \mathcal{M}^{X}, x \in X
\end{aligned}
$$

(H2) $\Lambda: \mathbb{R}_{+}^{X} \rightarrow \mathbb{R}_{+}^{X}$ is defined by

$$
(\Lambda \delta)(x):=|\lambda| \delta(p(x))+|\nu| \delta(q(x)), \quad \delta \in \mathbb{R}_{+}^{X}, x \in X
$$

The subsequent theorem follows easily from [7, Theorem 1].

TheOREM 2.3. Assume that hypotheses (H1) and (H2) are valid and there are $\varepsilon: X \rightarrow \mathbb{R}_{+}$and $\varphi: X \rightarrow \mathcal{M}$ with

$$
\begin{array}{ll}
\|(\mathcal{T} \varphi)(x)-\varphi(x)\| \leq \varepsilon(x), & x \in X, \\
\varepsilon^{*}(x):=\sum_{n=0}^{\infty} \Lambda^{n} \varepsilon(x)<\infty, & x \in X .
\end{array}
$$

Then there exists a unique fixed point $\psi$ of $\mathcal{T}$ such that

$$
\|\varphi(x)-\psi(x)\| \leq \varepsilon^{*}(x), \quad x \in X .
$$

Moreover,

$$
\psi(x):=\lim _{n \rightarrow \infty}\left(\mathcal{T}^{n} \varphi\right)(x), \quad x \in X .
$$

Let us recall that $\mathcal{I} \subset 2^{\mathcal{A}}$ is an ideal provided $2^{D} \subset \mathcal{I}$ and $C \cup D \in \mathcal{I}$ for every $C, D \in \mathcal{I}$. Next, we write $D+x:=\{x+y: y \in D\}$ and $\gamma D:=\{\gamma y$ : $y \in D\}$ for $x \in \mathcal{A}, \gamma \in \mathbb{F}$, and $D \in 2^{\mathcal{A}}$. We will need the following.

Lemma 2.4 ([6, Proposition 3.8]). Assume that $\mathcal{I} \subset 2^{\mathcal{A}}$ is an ideal such that

$$
D+x \in \mathcal{I}, \quad D \in \mathcal{I}, x \in \mathcal{A} \text {. }
$$

If $\mathcal{I} \neq 2^{\mathcal{A}}, B \in \mathcal{I}$, and $h: \mathcal{A} \backslash B \rightarrow \mathcal{M}$ satisfies

$$
h(x+y)=h(x)+h(y), \quad x, y \in \mathcal{A} \backslash B, x+y \in \mathcal{A} \backslash B,
$$

then there is a unique additive $f: \mathcal{A} \rightarrow \mathcal{M}$ with $h(x)=f(x)$ for $x \in \mathcal{A} \backslash B$.

Remark 2.5. Clearly, if $\mathcal{I}=\{D \subset \mathcal{A}: \operatorname{card} D<\operatorname{card} \mathcal{A}\}$, then $\mathcal{I}$ is an ideal satisfying (2.1) and, in particular, $\{x\} \in \mathcal{I}$ for each $x \in \mathcal{A}$. The next natural examples of such ideals are given below.

(a) $\mathcal{I}=\{D \subset \mathcal{A}: \sup h(D)<\infty\}$ for some additive and nontrivial $h$ : $\mathcal{A} \rightarrow \mathbb{R}$. 
(b) $\mathcal{A}$ is a real normed space and $\mathcal{I}=\{D \subset \mathcal{A}: D$ is bounded $\}$.

(c) $\mathcal{A}$ is a real topological linear space of the second category of Baire and $\mathcal{I}$ is the family of all first category subsets of $\mathcal{A}$.

(d) $\mathcal{A}$ is a locally compact real linear topological space and $\mathcal{I}=\{A \subset \mathcal{A}$ : $\mu(A)<\infty\}$, where $\mu$ denotes the Haar measure in $\mathcal{A}$.

(e) $\mathcal{A}$ is a Polish linear space and $\mathcal{I}$ is the $\sigma$-ideal of Christensen zero subsets of $\mathcal{A}$ (see [9]).

In what follows $\mathcal{I} \subset 2^{\mathcal{A}}$ always denotes an ideal, which is proper (i.e., $\mathcal{I} \neq 2^{\mathcal{A}}$ ) and satisfies the condition (2.1).

We are now in a position to prove our main auxiliary result.

Theorem 2.6. Let $\Gamma \subset \mathbb{F}, B \in \mathcal{I}, A:=\mathcal{A} \backslash B, \psi: A^{2} \rightarrow[0, \infty)$, $d: A \rightarrow \mathcal{M}$, and

(2.2) $\|d(\gamma x+y)-\gamma d(x)-d(y)\| \leq \psi(x, y), \quad x, y \in A, \gamma \in \Gamma, \gamma x+y \in A$.

Suppose that one of the following two collections of assumptions is fulfilled.

(i) There are $\xi: A^{2} \rightarrow[0,1), c \in \mathbb{F} \backslash\{0\}$, and $\mu \in \Gamma \backslash\{0\}$ such that $c A \subset \mu A,(1-c) A \subset A$ and

$$
\begin{gathered}
|\mu| \psi\left(\frac{c}{\mu} x, \frac{c}{\mu} y\right)+\psi((1-c) x,(1-c) y) \\
\leq \xi(x, y) \psi(x, y), \quad x, y \in A, \\
\xi\left(\frac{c}{\mu} x, \frac{c}{\mu} y\right) \leq \xi(x, y), \quad \xi((1-c) x,(1-c) y) \leq \xi(x, y), \quad x, y \in A .
\end{gathered}
$$

(ii) There is $\mu \in \Gamma \backslash\{0,-1\}$ such that $A \subset(\mu+1) A$,

$$
\begin{gathered}
\bar{\psi}(x):=\sum_{n=0}^{\infty}|\mu+1|^{n} \psi\left((\mu+1)^{-n} x,(\mu+1)^{-n} x\right)<\infty, \quad x \in A, \\
\lim _{n \rightarrow \infty}|\mu+1|^{n} \psi\left((\mu+1)^{-n} x,(\mu+1)^{-n} y\right)=0, \quad x, y \in A .
\end{gathered}
$$

Then there exists a unique additive mapping $D: \mathcal{A} \rightarrow \mathcal{M}$ such that

$$
\begin{aligned}
& D(\gamma x)=\gamma D(x), \quad x \in \mathcal{A}, \gamma \in \Gamma, \\
& \|d(x)-D(x)\| \leq \Psi(x), \quad x \in A,
\end{aligned}
$$

where, for each $x \in A$,

$$
\Psi(x)=\left\{\begin{array}{ll}
(1-\xi((c / \mu) x,(1-c) x))^{-1} \psi((c / \mu) x,(1-c) x), & \text { if (i) holds } \\
\bar{\psi}\left((\mu+1)^{-1} x\right), & \text { if (ii) holds }
\end{array} .\right.
$$

In particular,

$$
D(x):=\lim _{n \rightarrow \infty}\left(\mathcal{T}^{n} d\right)(x), \quad x \in A,
$$

with $\mathcal{T}$ defined by (2.16). Moreover, if $\Gamma$ has a bounded subset belonging to $\mathfrak{B}_{\mathbb{F}}$, then $D$ is $\mathbb{F}$-linear. 
Proof. Let

$$
\lambda:=\left\{\begin{array}{ll}
\mu, & \text { if (i) holds } \\
\mu+1, & \text { if (ii) holds }
\end{array}, \quad \nu:=\left\{\begin{array}{ll}
1, & \text { if (i) holds } \\
0, & \text { if (ii) holds }
\end{array} .\right.\right.
$$

Given $a \in \mathbb{F}$, we define $\Lambda_{a}: \mathbb{R}_{+}^{A^{2}} \rightarrow \mathbb{R}_{+}^{A^{2}}$ by

$$
\Lambda_{a} \delta(x, y):=|\lambda| \delta\left(\frac{a}{\mu} x, \frac{a}{\mu} y\right)+\nu \delta((1-a) x,(1-a) y)
$$

for $\delta \in \mathbb{R}_{+}^{A^{2}},(x, y) \in A^{2}$, and $\psi_{a}: A^{2} \rightarrow[0, \infty)$ by

$$
\psi_{a}(x, y):=\psi\left(\frac{a}{\mu} x,(1-a) y\right), \quad x, y \in A .
$$

Note that in the case when (i) holds, by (2.3), we have

$$
\Lambda_{c} \psi(x, y) \leq \xi(x, y) \psi(x, y), \quad x, y \in A,
$$

whence, by induction, in view of (2.4), we easily get

$$
\left(\Lambda_{c}\right)^{n} \psi(x, y) \leq \xi(x, y)^{n} \psi(x, y), \quad x, y \in A, n \in \mathbb{N},
$$

where $\mathbb{N}$ denotes the set of positive integers. Consequently,

$$
\begin{gathered}
\sum_{n=0}^{\infty}\left(\Lambda_{c}\right)^{n} \psi_{c}(x, x) \leq \frac{\psi_{c}(x, x)}{1-\xi((c / \mu) x,(1-c) x)}, \quad x \in A, \\
\lim _{n \rightarrow \infty}\left(\Lambda_{c}\right)^{n} \psi(x, y)=0, \quad x, y \in A .
\end{gathered}
$$

On the other hand, in the case of (ii), (2.5) and (2.6) mean that (2.10) holds with $c=\mu /(\mu+1)$ and

$$
\sum_{n=0}^{\infty}\left(\Lambda_{\mu /(\mu+1)}\right)^{n} \psi_{\mu /(\mu+1)}(x, x)<\infty, \quad x \in A,
$$

because $\psi_{\mu /(\mu+1)}(x, x)=\psi\left((\mu+1)^{-1} x,(\mu+1)^{-1} x\right)$ for $x \in A$. So, writing

$$
a:=\left\{\begin{array}{ll}
c, & \text { if (i) holds } \\
\mu /(\mu+1), & \text { if (ii) holds }
\end{array},\right.
$$

on account of $(2.6),(2.9),(2.10)$, and (2.11) we get

$$
\begin{aligned}
& \sum_{n=0}^{\infty}\left(\Lambda_{a}\right)^{n} \psi_{a}(x, x)<\infty, \quad x \in A, \\
& \lim _{n \rightarrow \infty}\left(\Lambda_{a}\right)^{n} \psi(x, y)=0, \quad x, y \in A .
\end{aligned}
$$

In the rest of the proof we assume that $a$ is described by (2.12). Replacing $x$ by $(a / \mu) x$ and taking $\gamma=\mu, y=(1-a) x$ in $(2.2)$ we obtain the inequality

$$
\left\|d(x)-\mu d\left(\frac{a}{\mu} x\right)-d((1-a) x)\right\| \leq \psi\left(\frac{a}{\mu} x,(1-a) x\right), \quad x \in A .
$$


Now, let $\mathcal{T}: \mathcal{M}^{A} \rightarrow \mathcal{M}^{A}$ be defined by

$$
\mathcal{T} \eta(x):=\lambda \eta\left(\frac{a}{\mu} x\right)+\nu \eta((1-a) x), \quad x \in A, \eta \in \mathcal{M}^{A} .
$$

Then (H1) holds with $p(x)=(a / \mu) x$ and $q(x)=(1-a) x$ for $x \in A$. Using (2.15), we get

$$
\|\mathcal{T} d(x)-d(x)\| \leq \psi_{a}(x, x)=: \widetilde{\psi}(x), \quad x \in A .
$$

Next, $\Lambda_{a} \psi_{a}(x, x)=\Lambda \widetilde{\psi}(x)$ for $x \in A$, where $\Lambda$ is defined by (H2). Hence, according to (2.13) and Theorem 2.3 (with $\varepsilon=\widetilde{\psi}$ and $\varphi=d$ ), there is a fixed point $D_{0}: A \rightarrow \mathcal{M}$ of $\mathcal{T}$ such that

$$
\left\|d(x)-D_{0}(x)\right\| \leq \sum_{n=0}^{\infty} \Lambda^{n} \widetilde{\psi}(x)=\sum_{n=0}^{\infty}\left(\Lambda_{a}\right)^{n} \psi_{a}(x, x), \quad x \in A .
$$

Moreover $D_{0}(x):=\lim _{n \rightarrow \infty}\left(\mathcal{T}^{n} d\right)(x)$ for $x \in A$. Clearly, $D_{0}$ is a solution of the functional equation

$$
D(x)=\lambda D\left(\frac{a}{\mu} x\right)+\nu D((1-a) x) .
$$

Note that (2.9) (in the case of (i)) and (2.5) (in the case of (ii)) imply that

$$
\left\|D_{0}(x)-d(x)\right\| \leq \Psi(x), \quad x \in X .
$$

In the next step we show that for each $n \in \mathbb{N}_{0}:=\mathbb{N} \cup\{0\}$, we have

$$
\left\|\mathcal{T}^{n} d(\gamma x+y)-\gamma \mathcal{T}^{n} d(x)-\mathcal{T}^{n} d(y)\right\| \leq\left(\Lambda_{a}\right)^{n} \psi(x, y)
$$

for all $(x, y) \in A^{2}$ and $\gamma \in \Gamma$ with $\gamma x+y \in A$. Clearly, the case $n=0$ is trivial. So, fix $m \in \mathbb{N}_{0}$ and assume that (2.19) is true for $n=m$. Then

$$
\begin{array}{rl}
\| \mathcal{T}^{m+1} & d(\gamma x+y)-\gamma \mathcal{T}^{m+1} d(x)-\mathcal{T}^{m+1} d(y) \| \\
= & \| \lambda \mathcal{T}^{m} d\left(\frac{a}{\mu}(\gamma x+y)\right)+\nu \mathcal{T}^{m} d((1-a)(\gamma x+y)) \\
& -\gamma \lambda \mathcal{T}^{m} d\left(\frac{a}{\mu} x\right)-\gamma \nu \mathcal{T}^{m} d((1-a) x) \\
& -\lambda \mathcal{T}^{m} d\left(\frac{a}{\mu} y\right)-\nu \mathcal{T}^{m} d((1-a) y) \| \\
\leq & |\lambda|\left\|\mathcal{T}^{m} d\left(\frac{a}{\mu}(\gamma x+y)\right)-\gamma \mathcal{T}^{m} d\left(\frac{a}{\mu} x\right)-\mathcal{T}^{m} d\left(\frac{a}{\mu} y\right)\right\| \\
& +\nu\left\|\mathcal{T}^{m} d((1-a)(\gamma x+y))-\gamma \mathcal{T}^{m} d((1-a) x)-\mathcal{T}^{m} d((1-a) y)\right\| \\
\leq & |\lambda|\left(\Lambda_{a}\right)^{m} \psi\left(\frac{a}{\mu} x, \frac{a}{\mu} y\right)+\nu\left(\Lambda_{a}\right)^{m} \psi((1-a) x,(1-a) y) \\
= & \left(\Lambda_{a}\right)^{m+1} \psi(x, y), \quad x, y \in A, \gamma \in \Gamma, \gamma x+y \in A .
\end{array}
$$


Thus, by induction, we have shown that (2.19) holds for all $x, y \in A$ and $\gamma \in \Gamma$ with $\gamma x+y \in A$ and all $n \in \mathbb{N}_{0}$. Taking the limit $n \rightarrow \infty$ in inequality (20), due to identity (2.14), we deduce that

$$
D_{0}(\gamma x+y)=\gamma D_{0}(x)+D_{0}(y), \quad x, y \in A, \gamma \in \Gamma, \gamma x+y \in A .
$$

Take $x, y \in A$ with $x+y \in A$ and $\gamma \in \Gamma \backslash\{0\}$. Since $\mathcal{I}$ is an ideal satisfying (2.1), there is $z \in A \cap(A-\gamma y) \cap(A-\gamma(x+y))$. Clearly, $\gamma y+z, \gamma(x+y)+z \in A$ and, by (2.20),

$$
\begin{array}{r}
\gamma D_{0}(x)+\gamma D_{0}(y)+D_{0}(z)=\gamma D_{0}(x)+D_{0}(\gamma y+z) \\
=D_{0}(\gamma(x+y)+z)=\gamma D_{0}(x+y)+D_{0}(z) .
\end{array}
$$

In this way we have proved that

$$
D_{0}(x+y)=D_{0}(x)+D_{0}(y), \quad x, y \in A, x+y \in A .
$$

Hence, by Lemma 2.4, there exists an additive $D: \mathcal{A} \rightarrow \mathcal{M}$ such that $D_{0}(x)=$ $D(x)$ for $x \in A$.

Let $x \in \mathcal{A}$ and $\gamma \in \Gamma \backslash\{0\}$. Clearly $A \cap(x+A) \neq \emptyset$, which means that $x=x_{1}-x_{2}$ with some $x_{1}, x_{2} \in A$. Take

$$
y \in A \cap\left(A+\gamma x_{2}\right) \cap\left(A+\gamma x_{2}-\gamma x_{1}\right) .
$$

Using (2.20) and the additivity of $D$ we derive

$$
\begin{aligned}
\gamma D(x)+D(y) & =\gamma D\left(x_{1}\right)+D(y)-\gamma D\left(-x_{2}\right) \\
& =\gamma D\left(x_{1}\right)+D\left(-\gamma x_{2}+y\right) \\
& =D\left(\gamma x_{1}-\gamma x_{2}+y\right) \\
& =D(\gamma x)+D(y) .
\end{aligned}
$$

Thus, we have proved (2.7). If $\Gamma$ has a bounded subset belonging to $\mathfrak{B}_{\mathbb{F}}$, then Lemma 2.1 implies that $D$ is $\mathbb{F}$-linear.

To prove the uniqueness part of the theorem, assume that there exists an additive mapping $\widetilde{D}: \mathcal{A} \rightarrow \mathcal{M}$ with the properties

$$
\widetilde{D}(\mu x)=\mu \widetilde{D}(x), \quad\|d(x)-\widetilde{D}(x)\| \leq \Psi(x), \quad x \in A .
$$

Then it is easy to see that $\widetilde{D}$ is a solution of the equation (2.17) (i.e., it is a fixed point of $\mathcal{T}$ ) and

$$
\|D(x)-\widetilde{D}(x)\| \leq 2 \Psi(x), \quad x \in A
$$

We have to consider the cases (i) and (ii) separately.

Case (i). We have

$$
\Psi(x)=\frac{\psi((c / \mu) x,(1-c) x)}{1-\xi((c / \mu) x,(1-c) x)}, \quad x \in A .
$$


We show by induction that, for each $j \in \mathbb{N}_{0}$,

$$
\|D(x)-\widetilde{D}(x)\| \leq 2 \frac{\left(\Lambda_{a}\right)^{j} \psi((c / \mu) x,(1-c) x)}{1-\xi((c / \mu) x,(1-c) x)}, \quad x \in A .
$$

The case $j=0$ is exactly (2.22). So, fix $j \in \mathbb{N}_{0}$ and assume that (2.23) holds for $j$. Then, by (2.4), we get

$$
\begin{aligned}
\| D(x) & -\widetilde{D}(x)\|=\| \mathcal{T} D(x)-\mathcal{T} \widetilde{D}(x) \| \\
& =\left\|\lambda D\left(\frac{c}{\mu} x\right)+\nu D((1-c) x)-\lambda \widetilde{D}\left(\frac{c}{\mu} x\right)-\nu \widetilde{D}((1-c))\right\| \\
& \leq 2|\lambda| \frac{\left(\Lambda_{a}\right)^{j} \psi\left((c / \mu)^{2} x,(1-c)(c / \mu) x\right)}{1-\xi\left((c / \mu)^{2} x,(1-c)(c / \mu) x\right)} \\
& +2|\nu| \frac{\left(\Lambda_{a}\right)^{j} \psi\left((c / \mu)(1-c) x,(1-c)^{2} x\right)}{1-\xi\left((c / \mu)(1-c) x,(1-c)^{2} x\right)} \\
& \leq 2 \frac{|\lambda|\left(\Lambda_{a}\right)^{j} \psi\left((c / \mu)^{2} x,(1-c)(c / \mu) x\right)}{1-\xi((c / \mu) x,(1-c) x)} \\
& +2 \frac{|\nu|\left(\Lambda_{a}\right)^{j} \psi\left((c / \mu)(1-c) x,(1-c)^{2} x\right)}{1-\xi((c / \mu) x,(1-c) x)} \\
& =2 \frac{\left(\Lambda_{a}\right)^{j+1} \psi((c / \mu) x,(1-c) x)}{1-\xi((c / \mu) x,(1-c) x)}, \quad x \in A .
\end{aligned}
$$

Thus, we have shown (2.23). Letting $j \rightarrow \infty$ in (2.23) and using (2.14), we get $\widetilde{D}(x)=D(x)$ for $x \in A$, whence Lemma 2.4 yields $\widetilde{D}=D$.

CAsE (ii). In this case

$$
\Psi(x)=\sum_{n=0}^{\infty}|\mu+1|^{n} \psi\left(\frac{1}{(\mu+1)^{n+1}} x, \frac{1}{(\mu+1)^{n+1}} x\right), \quad x \in A .
$$

We show that, for every $j \in \mathbb{N}_{0}$ and $x \in A$, we have

$$
\|D(x)-\widetilde{D}(x)\| \leq 2 \sum_{n=j}^{\infty}|\mu+1|^{n} \psi\left(\frac{1}{(\mu+1)^{n+1}} x, \frac{1}{(\mu+1)^{n+1}} x\right)
$$


The case $j=0$ is exactly (2.22). So, fix $j \in \mathbb{N}_{0}$ and assume that (2.24) holds for $j$. Then, for each $x \in A$, we get

$$
\begin{aligned}
\| D(x) & -\widetilde{D}(x)\|=\| \mathcal{T} D(x)-\mathcal{T} \widetilde{D}(x) \| \\
& =\left\|(\mu+1) D\left(\frac{1}{\mu+1} x\right)-(\mu+1) \widetilde{D}\left(\frac{1}{\mu+1} x\right)\right\| \\
& \leq 2|\mu+1| \sum_{n=j}^{\infty}|\mu+1|^{n} \psi\left(\frac{1}{(\mu+1)^{n+2}} x, \frac{1}{(\mu+1)^{n+2}} x\right) \\
& =2 \sum_{n=j+1}^{\infty}|\mu+1|^{n} \psi\left(\frac{1}{(\mu+1)^{n+1}} x, \frac{1}{(\mu+1)^{n+1}} x\right) .
\end{aligned}
$$

Thus, we have proved (2.24). Letting $j \rightarrow \infty$ in (2.24), due to assumption (2.6) we get that $\widetilde{D}(x)=D(x)$ for $x \in A$, whence, by Lemma 2.4, we have $\widetilde{D}=D$. The proof is completed.

The next theorem provides a result that is complementary to Theorem 2.6 (ii). We can prove it analogously as Theorem 2.6 (ii), but, for the convenience of the readers, we present a more direct, elementary, and simpler reasoning, patterned on the original ideas of Hyers from [13].

Theorem 2.7. Let $\Gamma \subset \mathbb{F}, B \in \mathcal{I}, A:=\mathcal{A} \backslash B, \psi: A^{2} \rightarrow[0, \infty)$, and let $d: \mathcal{A} \rightarrow \mathcal{M}$ satisfies the inequality (2.2). Suppose that there is $\mu \in \Gamma \backslash\{-1,0\}$ such that $(\mu+1) A \subset A$ and

$$
\begin{aligned}
& \bar{\psi}(x):=\sum_{n=0}^{\infty} \frac{\psi\left((\mu+1)^{n} x,(\mu+1)^{n} x\right)}{|\mu+1|^{n+1}}<\infty, \quad x \in A, \\
& \liminf _{n \rightarrow \infty} \frac{\psi\left((\mu+1)^{n} x,(\mu+1)^{n} y\right)}{|\mu+1|^{n}}=0, \quad x, y \in A .
\end{aligned}
$$

Then there exists a unique additive $D: \mathcal{A} \rightarrow \mathcal{M}$ such that (2.7) holds and

$$
\|d(x)-D(x)\| \leq \bar{\psi}(x), \quad x \in A .
$$

In particular,

$$
D(x)=\lim _{n \rightarrow \infty} \frac{d\left((\mu+1)^{n} x\right)}{(\mu+1)^{n}}, \quad x \in A .
$$

Moreover, if $\Gamma$ has a bounded subset belonging to $\mathfrak{B}_{\mathbb{F}}$, then $D$ is $\mathbb{F}$-linear.

Proof. Replacing $y$ by $x$ in (2.2), we obtain

$$
\|d((\gamma+1) x)-(\gamma+1) d(x)\| \leq \psi(x, x), \quad x \in A, \gamma \in \Gamma \backslash\{-1,0\},
$$

which, with $\gamma=\mu$, gives

$$
\left\|\frac{1}{\mu+1} d((\mu+1) x)-d(x)\right\| \leq \frac{\psi(x, x)}{|\mu+1|}, \quad x \in A .
$$


Using the induction, it is easy to see that

$$
\left\|\frac{d\left((\mu+1)^{p} x\right)}{(\mu+1)^{p}}-\frac{d\left((\mu+1)^{q} x\right)}{(\mu+1)^{q}}\right\| \leq \sum_{k=q}^{p-1} \frac{\psi\left((\mu+1)^{k} x,(\mu+1)^{k} x\right)}{|\mu+1|^{k+1}}
$$

for all $x \in A$ and all $p>q \geq 0$. By (2.25), it follows that for all $x \in A$, the sequence

$$
\left\{\frac{d\left((\mu+1)^{n} x\right)}{(\mu+1)^{n}}\right\}_{n=0}^{\infty}
$$

is Cauchy and, since $\mathcal{M}$ is complete, it is convergent. Thus, we can define a mapping $D_{0}: A \rightarrow \mathcal{M}$ by

$$
D_{0}(x)=\lim _{n \rightarrow \infty} \frac{d\left((\mu+1)^{n} x\right)}{(\mu+1)^{n}}, \quad x \in A
$$

Replacing $x$ by $(\mu+1)^{n} x$ and $y$ by $(\mu+1)^{n} y$ in $(2.2)$, we obtain

$$
\begin{array}{r}
\left\|\frac{d\left(\gamma(\mu+1)^{n} x+(\mu+1)^{n} y\right)}{(\mu+1)^{n}}-\gamma \frac{d\left((\mu+1)^{n} x\right)}{(\mu+1)^{n}}-\frac{d\left((\mu+1)^{n} y\right)}{(\mu+1)^{n}}\right\| \\
\leq \frac{\psi\left((\mu+1)^{n} x,(\mu+1)^{n} y\right)}{|\mu+1|^{n}}
\end{array}
$$

for $x, y \in A, \gamma \in \Gamma$ with $\gamma x+y \in A$ and $n \in \mathbb{N}$. Hence, by (2.26), we get (2.20). Next, in the same way as in the proof of Theorem 2.7, we deduce from (2.20) that there exists an additive $D: \mathcal{A} \rightarrow \mathcal{M}$ such that $D_{0}(x)=D(x)$ for $x \in A$ and (2.7) holds. Moreover, such $D$ is $\mathbb{F}$-linear when $\Gamma$ has a bounded subset belonging to $\mathfrak{B}_{\mathbb{F}}$.

Now, writing $q=0$ in $(2.27)$, we obtain

$$
\left\|\frac{d\left((\mu+1)^{p} x\right)}{(\mu+1)^{p}}-d(x)\right\| \leq \sum_{k=0}^{p-1} \frac{\psi\left((\mu+1)^{k} x,(\mu+1)^{k} x\right)}{|\mu+1|^{k+1}}, \quad x \in A,
$$

whence, letting $p \rightarrow \infty$, we get $\|d(x)-D(x)\| \leq \bar{\psi}(x)$ for $x \in A$.

It remains to show that $D$ is the unique additive mapping satisfying (2.7). So, suppose that there exists another additive mapping $\widetilde{D}: \mathcal{A} \rightarrow \mathcal{M}$ such that $\widetilde{D}(\gamma x)=\gamma \widetilde{D}(x)$ for $x \in \mathcal{A}, \gamma \in \Gamma$, and $\|d(x)-\widetilde{D}(x)\| \leq \bar{\psi}(x)$ for $x \in A$. Then it is easily seen that

$$
\widetilde{D}(x)=\frac{\widetilde{D}\left((\mu+1)^{n} x\right)}{(\mu+1)^{n}}, \quad x \in \mathcal{A}, n \in \mathbb{N},
$$


and, consequently, for all $x \in A$,

$$
\begin{aligned}
\|D(x)-\widetilde{D}(x)\| & =\lim _{n \rightarrow \infty}|\mu+1|^{-n}\left\|d\left((\mu+1)^{n} x\right)-\widetilde{D}\left((\mu+1)^{n} x\right)\right\| \\
& \leq \lim _{n \rightarrow \infty}|\mu+1|^{-n} \bar{\psi}\left((\mu+1)^{n} x\right) \\
& \leq \lim _{n \rightarrow \infty} \sum_{k=0}^{\infty} \frac{\psi\left((\mu+1)^{k+n} x,(\mu+1)^{k+n} x\right)}{|\mu+1|^{k+n+1}} \\
& =\lim _{n \rightarrow \infty} \sum_{k=n}^{\infty} \frac{\psi\left((\mu+1)^{k} x,(\mu+1)^{k} x\right)}{|\mu+1|^{k+1}}=0 .
\end{aligned}
$$

Therefore, $D(x)=\widetilde{D}(x)$ for $x \in A$ and, by Lemma $2.4, \widetilde{D}=D$. This completes the proof.

\section{Stability of Generalized Lie Derivations}

Given $A \subset \mathcal{A}$, we write $\widetilde{A}_{0}:=A \cup\{[x, y]: x, y \in A\}, \widetilde{A}:=\widetilde{A}_{0} \backslash\{0\}$ and, for $a \in \mathcal{A} \backslash\{0\}$ and $\gamma \in \mathbb{F} \backslash\{0\}$ with $a A \subset \gamma A$ and $(1-a) A \subset A$, we define a linear operator $\widehat{\Lambda}_{a}^{\gamma}: \mathbb{R}_{+}^{A^{2}} \cup \mathbb{R}_{+}^{\widetilde{A}^{2}} \rightarrow \mathbb{R}_{+}^{A^{2}} \cup \mathbb{R}_{+}^{\widetilde{A}^{2}}$ by

$$
\begin{array}{r}
\widehat{\Lambda} \widehat{\Lambda}_{a}^{\gamma} \delta(x, y):=|\gamma|^{2} \delta\left(\frac{a}{\gamma} x, \frac{a}{\gamma} y\right)+|\gamma| \delta\left(\frac{a}{\gamma} x,(1-a) y\right)+|\gamma| \delta\left((1-a) x, \frac{a}{\gamma} y\right) \\
+\delta((1-a) x,(1-a) y), \quad \delta \in \mathbb{R}_{+}^{A^{2}} \cup \mathbb{R}_{+}^{\widetilde{A}^{2}}, x, y \in A .
\end{array}
$$

The next two theorems are the main results of this paper.

Theorem 3.1. Let $\Gamma \subset \mathbb{F}, B \in \mathcal{I}, A:=\mathcal{A} \backslash B, d: A \rightarrow \mathcal{M}, g: \widetilde{A}_{0} \rightarrow \mathcal{M}$, and $\varphi_{1}, \varphi_{2}, \varphi_{3}: \widetilde{A}^{2} \rightarrow[0, \infty)$ satisfy the following three inequalities

(3.1) $\|d(\gamma x+y)-\gamma d(x)-d(y)\| \leq \varphi_{1}(x, y), \quad x, y \in A, \gamma \in \Gamma, \gamma x+y \in A$,

(3.2) $\|g(\gamma x+y)-\gamma g(x)-g(y)\| \leq \varphi_{2}(x, y), \quad x, y \in \widetilde{A}, \gamma \in \Gamma, \gamma x+y \in \widetilde{A}$,

(3.3) $\|g([x, y])-g(x) y+g(y) x-x d(y)+y d(x)\| \leq \varphi_{3}(x, y), \quad x, y \in A$.

Suppose that one of the following two collections of assumptions is fulfilled.

(i) There are $\xi_{1}, \xi_{2}: \widetilde{A}^{2} \rightarrow[0,1), c \in \mathbb{F} \backslash\{0\}, \mu \in \Gamma \backslash\{0\}$ such that $c A \subset \mu A,(1-c) A \subset A$ and, for every $x, y \in \widetilde{A}, j=1,2$,

$$
\begin{gathered}
|\mu| \varphi_{j}\left(\frac{c}{\mu} x, \frac{c}{\mu} y\right)+\varphi_{j}((1-c) x,(1-c) y) \leq \xi_{j}(x, y) \varphi_{j}(x, y), \\
\xi_{j}\left(\frac{c}{\mu} x, \frac{c}{\mu} y\right) \leq \xi_{j}(x, y), \quad \xi_{j}((1-c) x,(1-c) y) \leq \xi_{j}(x, y), \\
\lim _{n \rightarrow \infty}\left(\widehat{\Lambda}_{c}^{\mu}\right)^{n} \varphi_{3}(x, y)=0 .
\end{gathered}
$$


(ii) There is $\mu \in \Gamma \backslash\{0,-1\}$ with $A \subset(\mu+1) A$ and, for all $x, y \in \widetilde{A}$,

$$
\begin{gathered}
\bar{\varphi}_{j}(x)=\sum_{n=0}^{\infty}|\mu+1|^{n} \varphi_{j}\left((\mu+1)^{-n} x,(\mu+1)^{-n} x\right)<\infty \\
\lim _{n \rightarrow \infty}|\mu+1|^{n} \varphi_{j}\left((\mu+1)^{-n} x,(\mu+1)^{-n} y\right)=0 \\
\lim _{n \rightarrow \infty}|\mu+1|^{2 n} \varphi_{3}\left((\mu+1)^{-n} x,(\mu+1)^{-n} y\right)=0
\end{gathered}
$$

Then there exist a unique additive $D: \mathcal{A} \rightarrow \mathcal{M}$ and a unique generalized Lie $D$-derivation $G: \mathcal{A} \rightarrow \mathcal{M}$ such that

$$
G(\gamma x)=\gamma G(x), \quad D(\gamma x)=\gamma D(x), \quad x \in \mathcal{A}, \gamma \in \Gamma,
$$

(3.11) $\|d(x)-D(x)\| \leq \Phi_{1}(x), \quad\|g(y)-G(y)\| \leq \Phi_{2}(y), \quad x \in A, y \in \widetilde{A}$,

where $\Phi_{1}: A \rightarrow \mathcal{M}$ and $\Phi_{2}: \widetilde{A} \rightarrow \mathcal{M}$ are given by

$$
\Phi_{j}(x)=\left\{\begin{array}{ll}
\left(1-\xi_{j}((c / \mu) x,(1-c) x)\right)^{-1} \varphi_{j}((c / \mu) x,(1-c) x), & \text { if (i) holds } \\
\bar{\varphi}_{j}\left((\mu+1)^{-1} x\right), & \text { if (ii) holds }
\end{array} .\right.
$$

In particular,

$$
D(x):=\lim _{n \rightarrow \infty}\left(\mathcal{T}^{n} d\right)(x), \quad G(y):=\lim _{n \rightarrow \infty}\left(\mathcal{T}^{n} g\right)(y), \quad x \in A, y \in \widetilde{A}
$$

where $\mathcal{T}$ is given by (3.13) if (i) holds and by (3.14) if (ii) holds. Moreover, if $\Gamma$ has a bounded subset belonging to $\mathfrak{B}_{\mathbb{F}}$, then $D$ and $G$ are $\mathbb{F}$-linear.

Proof. It is easily seen that, by (3.1), (3.2), and Theorem 2.6 with $\psi=\varphi_{i}$ for $i=1,2$, there exist unique additive functions $G, D: \mathcal{A} \rightarrow \mathcal{M}$ satisfying (3.10) and (3.11). Moreover, (3.12) holds. Here, in the case of (i),

$$
\mathcal{T} \eta(x) \equiv \mu \eta\left(\frac{c}{\mu} x\right)+\eta((1-c) x), \quad \eta \in \mathcal{M}^{A} \cup \mathcal{M}^{\widetilde{A}} \cup \mathcal{M}^{\widetilde{A}_{0}}
$$

and, in the case of (ii),

$$
\mathcal{T} \eta(x) \equiv(\mu+1) \eta\left(\frac{1}{\mu+1} x\right), \quad \eta \in \mathcal{M}^{A} \cup \mathcal{M}^{\widetilde{A}} \cup \mathcal{M}^{\widetilde{A}_{0}} .
$$

If $\Gamma$ has a bounded subset belonging to $\mathfrak{B}_{\mathbb{F}}$, then $G$ and $D$ are $\mathbb{F}$-linear.

Next, we show that

$$
G([x, y])=G(x) y-G(y) x+x D(y)-y D(x), \quad x, y \in A,[x, y] \neq 0 .
$$

At this part, we have to distinguish two cases according to (i) and (ii). CASE (i). First, we prove that for all $n \in \mathbb{N}_{0}$,

$$
\begin{array}{r}
\left\|\mathcal{T}^{2 n} g([x, y])-\mathcal{T}^{n} g(x) y+\mathcal{T}^{n} g(y) x-x \mathcal{T}^{n} d(y)+y \mathcal{T}^{n} d(x)\right\| \\
\leq\left(\widehat{\Lambda}_{c}^{\mu}\right)^{n} \varphi_{3}(z, w), \quad x, y \in A,[x, y] \neq 0 .
\end{array}
$$


Since (3.16) with $n=0$ is just (3.3), it is enough to observe that for every $n \in \mathbb{N}_{0}$ and $x, y, z, w \in A$ with $[x, y] \neq 0$,

$$
\begin{aligned}
\mathcal{T}^{2(n+1)} g([x, y])= & \mu \mathcal{T}^{2 n+1} g\left(\frac{c}{\mu}[x, y]\right)+\mathcal{T}^{2 n+1} g((1-c)[x, y]) \\
= & \mu \mathcal{T}^{2 n+1} g\left(\left[\frac{c}{\mu} x, y\right]\right)+\mathcal{T}^{2 n+1} g([(1-c) x, y]) \\
= & \mu^{2} \mathcal{T}^{2 n} g\left(\left[\frac{c}{\mu} x, \frac{c}{\mu} y\right]\right)+\mu \mathcal{T}^{2 n} g\left(\left[\frac{c}{\mu} x,(1-c) y\right]\right) \\
& +\mu \mathcal{T}^{2 n} g\left(\left[(1-c) x, \frac{c}{\mu} y\right]\right)+\mathcal{T}^{2 n} g([(1-c) x,(1-c) y]), \\
\mathcal{T}^{n+1} g(z) w= & \mu^{2} \mathcal{T}^{n} g\left(\frac{c}{\mu} z\right) \frac{c}{\mu} w+\mu \mathcal{T}^{n} g\left(\frac{c}{\mu} z\right)(1-c) w \\
& +\mu \mathcal{T}^{n} g((1-c) z) \frac{c}{\mu} w+\mathcal{T}^{n} g((1-c) z)(1-c) w \\
w \mathcal{T}^{n+1} d(z)= & \mu^{2}\left(\frac{c}{\mu} w\right) \mathcal{T}^{n} d\left(\frac{c}{\mu} z\right)+\mu(1-c) w \mathcal{T}^{n} d\left(\frac{c}{\mu} z\right) \\
& +\mu\left(\frac{c}{\mu} w\right) \mathcal{T}^{n} d((1-c) z)+(1-c) w \mathcal{T}^{n} d((1-c) z) .
\end{aligned}
$$

Consequently, under the assumption that (3.16) holds for some $n \in \mathbb{N}_{0}$, we have

$$
\begin{aligned}
\left\|\mathcal{T}^{2(n+1)} g([x, y])-\mathcal{T}^{n+1} g(x) y+\mathcal{T}^{n+1} g(y) x-x \mathcal{T}^{n+1} d(y)+y \mathcal{T}^{n+1} d(x)\right\| \\
\leq|\mu|^{2}\left(\widehat{\Lambda}_{c}^{\mu}\right)^{n} \varphi_{3}\left(\frac{c}{\mu} x, \frac{c}{\mu} y\right)+|\mu|\left(\widehat{\Lambda}_{c}^{\mu}\right)^{n} \varphi_{3}\left(\frac{c}{\mu} x,(1-c) y\right) \\
\quad+|\mu|\left(\widehat{\Lambda}_{c}^{\mu}\right)^{n} \varphi_{3}\left((1-c) x, \frac{c}{\mu} y\right)+\left(\widehat{\Lambda}_{c}^{\mu}\right)^{n} \varphi_{3}((1-c) x,(1-c) y) \\
=\left(\widehat{\Lambda}_{c}^{\mu}\right)^{n+1} \varphi_{3}(x, y), \quad x, y \in A,[x, y] \neq 0, n \in \mathbb{N}_{0} .
\end{aligned}
$$

So, we have proved (3.16). Letting $n \rightarrow \infty$ in (3.16) and using (3.6), we obtain the equality (3.15), as desired.

CASE (ii). Note that

$$
\begin{array}{ll}
G(x)=\lim _{n \rightarrow \infty}(\mu+1)^{n} g\left((\mu+1)^{-n} x\right), & x \in \widetilde{A}, \\
D(x)=\lim _{n \rightarrow \infty}(\mu+1)^{n} d\left((\mu+1)^{-n} x\right), & x \in A .
\end{array}
$$


Hence, according to (3.3) and (3.9), for every $x, y \in A$ with $[x, y] \neq 0$, we have

$$
\begin{aligned}
\| G([x, y]) & -G(x) y+G(y) x-x D(y)+y D(x) \| \\
= & \lim _{n \rightarrow \infty}|\mu+1|^{2 n} \| g\left(\left[(\mu+1)^{-n} x,(\mu+1)^{-n} y\right]\right) \\
& -g\left((\mu+1)^{-n} x\right)(\mu+1)^{-n} y+g\left((\mu+1)^{-n} y\right)(\mu+1)^{-n} x \\
& -(\mu+1)^{-n} x d\left((\mu+1)^{-n} y\right)+(\mu+1)^{-n} y d\left((\mu+1)^{-n} x\right) \| \\
\leq & \lim _{n \rightarrow \infty}|\mu+1|^{2 n} \varphi_{3}\left((\mu+1)^{-n} x,(\mu+1)^{-n} y\right)=0 .
\end{aligned}
$$

Thus, (3.15) holds in this case as well.

Finally, let $z, w \in \mathcal{A}$ be arbitrary. Utilizing identity (3.15) and the additivity of the mappings $G$ and $D$, we receive

$$
\begin{aligned}
G([z, w])= & G\left(\left[z_{1}-z_{2}, w_{1}-w_{2}\right]\right) \\
= & G\left(\left[z_{1}, w_{1}\right]\right)-G\left(\left[z_{1}, w_{2}\right]\right)-G\left(\left[z_{2}, w_{1}\right]\right)+G\left(\left[z_{2}, w_{2}\right]\right) \\
= & G\left(z_{1}\right) w_{1}-G\left(w_{1}\right) z_{1}+z_{1} D\left(w_{1}\right)-w_{1} D\left(z_{1}\right) \\
& -G\left(z_{1}\right) w_{2}+G\left(w_{2}\right) z_{1}-z_{1} D\left(w_{2}\right)+w_{2} D\left(z_{1}\right) \\
& -G\left(z_{2}\right) w_{1}+G\left(w_{1}\right) z_{2}-z_{2} D\left(w_{1}\right)+w_{1} D\left(z_{2}\right) \\
& +G\left(z_{2}\right) w_{2}-G\left(w_{2}\right) z_{2}+z_{2} D\left(w_{2}\right)-w_{2} D\left(z_{2}\right) \\
= & G\left(z_{1}-z_{2}\right)\left(w_{1}-w_{2}\right)-G\left(w_{1}-w_{2}\right)\left(z_{1}-z_{2}\right) \\
& +\left(z_{1}-z_{2}\right) D\left(w_{1}-w_{2}\right)-\left(w_{1}-w_{2}\right) D\left(z_{1}-z_{2}\right) \\
= & G(z) w-G(w) z+z D(w)-w D(z) .
\end{aligned}
$$

In this way we have shown that $G$ is a generalized Lie $D$-derivation. The proof is completed.

As the following corollary shows, assumptions (3.2) and (3.3) in the previous theorem can be replaced by only one inequality (for the sake of the simplicity, we consider only the case $A=\mathcal{A}$ ).

Corollary 3.2. Let $\Gamma \subset \mathbb{F}, \varphi: \mathcal{A}^{4} \rightarrow[0, \infty), d, g: \mathcal{A} \rightarrow \mathcal{M}$ and

$$
\begin{aligned}
& \|d(\gamma x+y)-\gamma d(x)-d(y)\| \leq \varphi(x, y, 0,0), \quad x, y \in \mathcal{A}, \gamma \in \Gamma, \\
& \begin{array}{l}
\| g(\gamma x+y+[z, w])-\gamma g(x)-g(y)-g(z) w+g(w) z \\
\quad-z d(w)+w d(z) \| \leq \varphi(x, y, z, w), \quad x, y, z, w \in \mathcal{A}, \gamma \in \Gamma .
\end{array}
\end{aligned}
$$

Suppose that one of the collections of the assumptions (i) and (ii) of Theorem 3.1 is fulfilled for $A=\mathcal{A}, \varphi_{j}(x, y)=\varphi(x, y, 0,0)(x, y \in \mathcal{A}, j=1,2)$, and $\varphi_{3}(z, w)=\varphi(0,0, z, w)(z, w \in \mathcal{A})$. Then all the statements of Theorem 3.1 are valid with $A=\mathcal{A}$. 
Proof. Taking $z=w=0$ in (3.18) we get

$$
\|g(\gamma x+y)-\gamma g(x)-g(y)\| \leq \varphi_{2}(x, y), \quad x, y \in \mathcal{A}, \gamma \in \Gamma .
$$

Next, taking $x=y=0$ and $\gamma=\mu$ in (3.18), we obtain

$$
\begin{aligned}
& \| g([z, w])-g(z) w+g(w) z-z d(w)+w d(z) \| \\
& \leq \varphi_{3}(z, w)+|\mu+1|\|g(0)\|=: \widetilde{\varphi}_{3}(z, w), \quad z, w \in \mathcal{A} .
\end{aligned}
$$

Again, we observe the cases (i) and (ii) separately.

CAse (i). Clearly, (3.4) implies that

$$
(|\mu|+1) \varphi_{1}(0,0) \leq \xi(0,0) \varphi_{1}(0,0) .
$$

Since $|\mu|+1>1>\xi(0,0)$, this means that $\varphi_{2}(0,0)=0$. Consequently, (3.18) with $x=y=z=w=0$ and $\gamma=\mu$ yields $g(0)=0$. Hence, in this case $\varphi_{3}=\widetilde{\varphi}_{3}$ and it is enough to use Theorem 3.1 (i).

CASE (ii). Note that if $|\mu+1| \geq 1$, then from (3.8) we get $\varphi_{2}(0,0)=0$, whence (3.18), with $x=y=z=w=0$ and $\gamma=\mu$, yields $g(0)=0$. Consequently, $\varphi_{3}=\widetilde{\varphi}_{3}$ and it is enough to use Theorem 3.1 (ii).

Finally, when $|\mu+1|<1$, by (3.9), we have

$$
\begin{aligned}
\lim _{n \rightarrow \infty} & |\mu+1|^{n} \widetilde{\varphi}_{3}\left((\mu+1)^{-n} x,(\mu+1)^{-n} y\right) \\
= & \lim _{n \rightarrow \infty}|\mu+1|^{n} \varphi_{3}\left((\mu+1)^{-n} x,(\mu+1)^{-n} y\right) \\
& +\lim _{n \rightarrow \infty} \frac{|\mu+1|^{n+1}|| g(0) \mid}{1-|\mu+1|}=0, \quad x, y \in \mathcal{A} .
\end{aligned}
$$

Consequently, we use Theorem 3.1 (ii) with $\varphi_{3}$ replaced by $\widetilde{\varphi}_{3}$.

The next theorem is complementary to Theorem 3.1 (ii).

Theorem 3.3. Let $\Gamma \subset \mathbb{F}, B \in \mathcal{I}, A:=\mathcal{A} \backslash B, \varphi_{1}, \varphi_{2}, \varphi_{3}: \widetilde{A}^{2} \rightarrow[0, \infty)$, $d: A \rightarrow \mathcal{M}, g: \widetilde{A}_{0} \rightarrow \mathcal{M}$, and let (3.1)-(3.3) be valid. Suppose that there is $\mu \in \Gamma \backslash\{-1\}$ such that $(\mu+1) A \subset A$,

$$
\begin{gathered}
\bar{\varphi}_{i}(x):=\sum_{n=0}^{\infty} \frac{\varphi_{i}\left((\mu+1)^{n} x,(\mu+1)^{n} x\right)}{|\mu+1|^{n+1}}<\infty, \quad i=1,2, x \in \widetilde{A}, \\
\liminf _{n \rightarrow \infty} \frac{\varphi_{3}\left((\mu+1)^{n} x,(\mu+1)^{n} y\right)}{|\mu+1|^{2 n}}=0, \quad x, y \in A,
\end{gathered}
$$

and (2.26) is fulfilled by $\psi \in\left\{\varphi_{1}, \varphi_{2}\right\}$ with $A$ replaced by $\widetilde{A}$. Then there exist a unique additive $D: \mathcal{A} \rightarrow \mathcal{M}$ and a unique generalized Lie D-derivation $G: \mathcal{A} \rightarrow \mathcal{M}$ such that (3.10) holds and

(3.22) $\|d(x)-D(x)\| \leq \bar{\varphi}_{1}(x), \quad\|g(y)-G(y)\| \leq \bar{\varphi}_{2}(y), \quad x \in A, y \in \widetilde{A}$.

In particular, the condition (3.23) is valid. Moreover, if $\Gamma$ has a bounded subset belonging to $\mathfrak{B}_{\mathbb{F}}$, then $D$ and $G$ are $\mathbb{F}$-linear. 
Proof. According to (3.1), (3.2) and Theorem 2.7, there exist additive mappings $G, D: \mathcal{A} \rightarrow \mathcal{M}$ with

$$
D(x)=\lim _{n \rightarrow \infty} \frac{d\left((\mu+1)^{n} x\right)}{(\mu+1)^{n}}, \quad G(y)=\lim _{n \rightarrow \infty} \frac{g\left((\mu+1)^{n} y\right)}{(\mu+1)^{n}}
$$

for all $x \in A, y \in \widetilde{A}$. Moreover, the mappings $D$ and $G$ are the unique solutions of $(3.10)$, resp. (3.22). If $\Gamma$ has a bounded subset from $\mathfrak{B}_{\mathbb{F}}$, then $D$ and $G$ are $\mathbb{F}$-linear.

Further, for every $x, y \in A$,

$$
G([x, y])=\lim _{n \rightarrow \infty} \frac{g\left((\mu+1)^{2 n}[x, y]\right)}{(\mu+1)^{2 n}}=\frac{g\left(\left[(\mu+1)^{n} x,(\mu+1)^{n} y\right]\right)}{(\mu+1)^{2 n}},
$$

whence, in view of (3.3) and (3.21),

$$
\begin{aligned}
\| G([x, y]) & -G(x) y+G(y) x-x D(y)+y D(x) \| \\
= & \lim _{n \rightarrow \infty}|\mu+1|^{-2 n} \| g\left(\left[(\mu+1)^{n} x,(\mu+1)^{n} y\right]\right) \\
& -g\left((\mu+1)^{n} x\right)(\mu+1)^{n} y+g\left((\mu+1)^{n} y\right)(\mu+1)^{n} x \\
& -(\mu+1)^{n} x d\left((\mu+1)^{n} y\right)+(\mu+1)^{n} y d\left((\mu+1)^{n} x\right) \| \\
\leq & \liminf _{n \rightarrow \infty} \frac{\varphi_{3}\left((\mu+1)^{n} x,(\mu+1)^{n} y\right)}{|\mu+1|^{2 n}}=0 .
\end{aligned}
$$

Thus, we have obtained (3.15). Now, in the same way as at the very end of the proof of Theorem 3.1, we can show that $G$ is a generalized Lie $D$-derivation.

\section{Applichtions}

Let $A_{i} \subset \mathcal{A}, \eta_{i}, \varepsilon_{i} \in[0, \infty)$, and $p_{i} \in \mathbb{R}$ for $i=1,2,3$. Moreover, suppose that $p_{i} \geq 0$ or $0 \notin A_{i}$ for $i=1,2,3$. We will show applications of some of our results to a very simple case when $\mathcal{A}$ is a normed Lie algebra and

$$
\varphi_{i}(x, y):=\eta_{i}+\varepsilon_{i}\left(\|x\|^{p_{i}}+\|y\|^{p_{i}}\right), \quad x, y \in A_{i}, i=1,2,3 .
$$

In this section, we assume all the time that $\Gamma \subset \mathbb{F} \backslash\{0\}, \emptyset \neq A \subset \mathcal{A}$, $\varphi_{1}, \varphi_{2}, \varphi_{3}: \widetilde{A}^{2} \rightarrow[0, \infty)$ are defined by (4.1), and $g: \widetilde{A}_{0} \rightarrow \mathcal{M}$ and $d: A \rightarrow$ $\mathcal{M}$ are mappings satisfying conditions (3.1)-(3.3).

The first theorem reads as follows.

THEOREM 4.1. Let $p_{3} \neq 2, \eta_{3}\left(p_{3}-2\right) \leq 0$ and suppose that one of the following three conditions is valid

(a) $A=\mathcal{A}, \Gamma$ is unbounded, $p_{1}>1, p_{2}>1$, and $\eta_{1}=\eta_{2}=0$;

(b) $A=\mathcal{A} \backslash\{0\}, \Gamma$ is unbounded, and $p_{i}<1$ for $i=1,2$;

(c) $p_{i}<0$ for $i=1,2$ and there exists $B \in \mathcal{I}$ with $2 B=B$ and $A=\mathcal{A} \backslash B$. 
Then there exist a unique additive mapping $D: \mathcal{A} \rightarrow \mathcal{M}$ and a unique generalized Lie D-derivation $G: \mathcal{A} \rightarrow \mathcal{M}$ such that (3.10) holds and

$$
d(x)=D(x), \quad g(y)=G(y), \quad x \in A, y \in \widetilde{A} .
$$

If $\Gamma$ has a bounded subset from $\mathfrak{B}_{\mathbb{F}}$, then $G$ and $D$ are $\mathbb{F}$-linear.

Proof. Note that the condition $\eta_{3}\left(p_{3}-2\right) \leq 0$ is equivalent to the following one

$$
\text { if } p_{3}>2 \text {, then } \eta_{3}=0 \text {. }
$$

First, we show that there exist unique additive $D, G: \mathcal{A} \rightarrow \mathcal{M}$ fulfilling (3.10) and (4.2). We have to consider all three cases separately.

CAse (a). Clearly, the conditions (3.4) and (3.5) hold with the constant $\xi_{j}$ given by (4.15). Next, for each $n \in \mathbb{N}$, there are $\mu_{n} \in \Gamma$ and $c_{n} \in \mathbb{F}$ such that $\left|\mu_{n}\right| \geq n$,

$$
\left\|c_{n}\right\|^{p_{i}}\left|\mu_{n}\right|^{1-p_{i}}+\left\|1-c_{n}\right\|^{p_{i}}<1, \quad i=1,2,
$$

and $\lim _{n \rightarrow \infty} c_{n}=1$. Theorem 2.6 (i) implies that, for every $n \in \mathbb{N}$, there exist unique additive $D_{n}, G_{n}: \mathcal{A} \rightarrow \mathcal{M}$ such that

$$
\begin{gathered}
G_{n}(\gamma x)=\gamma G_{n}(x), \quad D_{n}(\gamma x)=\gamma D_{n}(x), \quad x \in \mathcal{A}, \gamma \in \Gamma, \\
\left\|d(x)-D_{n}(x)\right\| \leq \phi_{1, n}(x), \quad\left\|g(x)-G_{n}(x)\right\| \leq \phi_{2, n}(x), \quad x \in \mathcal{A},
\end{gathered}
$$

where

$$
\phi_{i, n}(x)=\frac{\left\|c_{n}\right\|^{p_{i}}\left|\mu_{n}\right|^{1-p_{i}}+\left\|1-c_{n}\right\|^{p_{i}}}{1-\left\|c_{n}\right\|^{p_{i}}\left|\mu_{n}\right|^{1-p_{i}}-\left\|1-c_{n}\right\|^{p_{i}}} \varepsilon_{i}\|x\|^{p_{i}}, \quad x \in \mathcal{A}, i=1,2 .
$$

The uniqueness of $D_{n}$ and $G_{n}$ implies that $D_{1}=D_{n}$ and $G_{1}=G_{n}$ for every $n \in \mathbb{N}$. Since

$$
\lim _{n \rightarrow \infty} \phi_{i, n}(x)=0, \quad x \in \mathcal{A},
$$

(4.5) yields (4.2) with $D=D_{1}$ and $G=G_{1}$.

CASE (b). For each $n \in \mathbb{N}$, there is $\mu_{n} \in \Gamma$ such that $\left|\mu_{n}+1\right| \geq n$. Note that

$$
\begin{aligned}
& \bar{\psi}_{i, n}(x):=\sum_{k=0}^{\infty} \frac{\varphi_{i}\left(\left(\mu_{n}+1\right)^{k} x,\left(\mu_{n}+1\right)^{k} x\right)}{\left|\mu_{n}+1\right|^{k+1}} \\
&=\frac{2}{\left|\mu_{n}+1\right|} \sum_{k=0}^{\infty}\left|\mu_{n}+1\right|^{k\left(p_{i}-1\right)} \varepsilon_{i} \|\left. x\right|^{p_{i}}+\sum_{k=0}^{\infty} \frac{\eta_{i}}{\left|\mu_{n}+1\right|^{k+1}} \\
&=\frac{2 \varepsilon_{i}\|x\|^{p_{i}}}{\left|\mu_{n}+1\right|-\left|\mu_{n}+1\right|^{p_{i}}}+\frac{\eta_{i}}{\left|\mu_{n}+1\right|-1}, \\
& \lim _{k \rightarrow \infty} \frac{\varphi_{i}\left(\left(\mu_{n}+1\right)^{k} x,\left(\mu_{n}+1\right)^{k} y\right)}{\left|\mu_{n}+1\right|^{k}}=\lim _{k \rightarrow \infty} \varepsilon_{i} \frac{\|x\|^{p_{i}}+\|y\|^{p_{i}}}{\left|\mu_{n}+1\right|^{k\left(1-p_{i}\right)}}+\frac{\eta_{i}}{\left|\mu_{n}+1\right|^{k}}=0,
\end{aligned}
$$


for $x, y \in A, n \in \mathbb{N}, i=1,2$. Hence, Theorem 2.7 implies that, for every $n \in \mathbb{N}$, there are unique additive $D_{n}, G_{n}: \mathcal{A} \rightarrow \mathcal{M}$ such that (4.4) holds and

$$
\left\|d(x)-D_{n}(x)\right\| \leq \bar{\psi}_{1, n}(x), \quad\left\|g(x)-G_{n}(x)\right\| \leq \bar{\psi}_{2, n}(x)(x), \quad x \in A .
$$

The uniqueness of $D_{n}$ and $G_{n}$ implies that $D_{1}=D_{n}$ and $G_{1}=G_{n}$ for every $n \in \mathbb{N}$. Since

$$
\lim _{n \rightarrow \infty} \bar{\psi}_{i, n}(x)=0, \quad x \in A, i=1,2,
$$

(4.6) yields (4.2) with $D=D_{1}$ and $G=G_{1}$.

CAsE (c). Fix $\mu \in \Gamma \backslash\{0\}$. Since $p_{1}<0$ and $p_{2}<0$, there is an $m \in \mathbb{N}$ such that

$$
2^{n p_{i}}|\mu|^{1-p_{i}}+\left(2^{n}-1\right)^{p_{i}}<1, \quad i=1,2, n \geq m .
$$

It is easy to check that conditions (2.3) and (2.4) hold with $\psi=\varphi_{i}$ and $\xi=\xi_{i, n}$ for $i=1,2$ and $n \in \mathbb{N}, n \geq m$, where

$$
\xi_{i, n}(x, y) \equiv 2^{n p_{i}}|\mu|^{1-p_{i}}+\left(2^{n}-1\right)^{p_{i}}, \quad i=1,2, n \in \mathbb{N}, n \geq m .
$$

From Theorem 2.6 (i) we derive that, for each $n \in \mathbb{N}, n \geq m$, there exist unique additive $D_{n}, G_{n}: \mathcal{A} \rightarrow \mathcal{M}$ such that (4.4) is fulfilled and

$$
\left\|d(x)-D_{n}(x)\right\| \leq \phi_{1, n}(x), \quad\left\|g(x)-G_{n}(y)\right\| \leq \phi_{2, n}(y)
$$

for $x \in A, y \in \widetilde{A}$ and with

$$
\phi_{i, n}(x)=\frac{2^{n p_{i}}|\mu|^{1-p_{i}}+\left(2^{n}-1\right)^{p_{i}}}{1-2^{n p_{i}}|\mu|^{1-p_{i}}+\left(2^{n}-1\right)^{p_{i}}} \varepsilon_{1}\|x\|^{p_{i}}
$$

for $i=1,2, n \in \mathbb{N}, n \geq m, x \in \widetilde{A}$. Clearly, the uniqueness of $D_{n}$ and $G_{n}$ implies that

$$
D_{m}=D_{n}, \quad G_{m}=G_{n}, \quad n \in \mathbb{N}, n \geq m .
$$

Consequently, (4.7) yields (4.2) for $D=D_{1}$ and $G=G_{1}$ because

$$
\lim _{n \rightarrow \infty} \phi_{i, n+m}(x)=0, \quad i=1,2, x \in \widetilde{A} .
$$

Thus, we have proved that, in all those three cases, there exist unique additive $D, G: \mathcal{A} \rightarrow \mathcal{M}$ such that (3.10) and (4.2) are valid. Moreover, if $\Gamma$ has a bounded subset belonging to $\mathfrak{B}_{\mathbb{F}}$, then (3.10) and Lemma 2.1 imply that $D$ and $G$ are $\mathbb{F}$-linear.

It remains to show that $G$ is a generalized Lie $D$-derivation. To this end, we first prove that

$$
\begin{aligned}
& g([x, y])=g(x) y-g(y) x+x d(y)-y d(x), \quad x, y \in A,[x, y] \neq 0, \\
& g(x) y-g(y) x+x d(y)-y d(x)=0, \quad x, y \in A,[x, y]=0 .
\end{aligned}
$$


Suppose that $p_{3}<2$ and fix $x, y \in A$. If $[x, y] \neq 0$, then, according to (3.3) and (4.2),

$$
\begin{aligned}
\| g([x, y]) & -g(x) y+g(y) x-x d(y)+y d(x) \| \\
= & 2^{-2 n} \| g\left(\left[2^{n} x, 2^{n} y\right]\right)-g\left(2^{n} x\right) 2^{n} y \\
& +g\left(2^{n} y\right) 2^{n} x-2^{n} x d\left(2^{n} y\right)+2^{n} y d\left(2^{n} x\right) \| \\
\leq & 2^{-2 n} \varepsilon_{3}\left(\left\|2^{n} x\right\|^{p_{3}}+\left\|2^{n} y\right\|^{p_{3}}\right)+2^{-2 n} \eta_{3} \\
= & 2^{\left(p_{3}-2\right) n} \varepsilon_{3}\left(\|x\|^{p_{3}}+\|y\|^{p_{3}}\right)+2^{-2 n} \eta_{3}, \quad n \in \mathbb{N},
\end{aligned}
$$

whence, letting $n \rightarrow \infty$, we obtain that (4.8) holds. If $[x, y]=0$, then for each $n \in \mathbb{N}$, we have $g(0)=g\left(\left[2^{n} x, 2^{n} y\right]\right)$ and, again by (3.3) and (4.2),

$$
\begin{aligned}
& \left\|g(0)-2^{2 n}(g(x) y-g(y) x+x d(y)-y d(x))\right\| \\
& \quad \leq \varepsilon_{3}\left(\left\|2^{n} x\right\|^{p_{3}}+\left\|2^{n} y\right\|^{p_{3}}\right)+\eta_{3}=2^{p_{3} n} \varepsilon_{3}\left(\|x\|^{p_{3}}+\|y\|^{p_{3}}\right)+\eta_{3},
\end{aligned}
$$

whence

$$
\begin{array}{r}
\left\|2^{-2 n} g(0)-g(x) y+g(y) x-x d(y)+y d(x)\right\| \\
\leq 2^{\left(p_{3}-2\right) n} \varepsilon_{3}\left(\|x\|^{p_{3}}+\|y\|^{p_{3}}\right)+2^{-2 n} \eta_{3},
\end{array}
$$

which, with $n \rightarrow \infty$, yields (4.9).

Now, let $p_{3}>2$ and $x, y \in A$. If $[x, y] \neq 0$, then, in view of (3.3), (4.2), and (4.3), we obtain that

$$
\begin{aligned}
\| g([x, y]) & -g(x) y+g(y) x-x d(y)+y d(x) \| \\
= & 2^{2 n} \| g\left(\left[2^{-n} x, 2^{-n} y\right]\right)-g\left(2^{-n} x\right) 2^{-n} y \\
& +g\left(2^{-n} y\right) 2^{-n} x-2^{-n} x d\left(2^{-n} y\right)+2^{-n} y d\left(2^{-n} x\right) \| \\
\leq & 2^{2 n} \varepsilon_{3}\left(\left\|2^{-n} x\right\|^{p_{3}}+\left\|2^{-n} y\right\|^{p_{3}}\right) \\
= & 2^{\left(2-p_{3}\right) n} \varepsilon_{3}\left(\|x\|^{p_{3}}+\|y\|^{p_{3}}\right), \quad n \in \mathbb{N} .
\end{aligned}
$$

Letting $n \rightarrow \infty$, we get (4.8). If $[x, y]=0$, then, again by (3.3), (4.2), and (4.3), for every $n \in \mathbb{N}$, we have

$$
\begin{aligned}
& \left\|g(0)-2^{-2 n}(g(x) y-g(y) x+x d(y)-y d(x))\right\| \\
& \quad \leq \varepsilon_{3}\left(\left\|2^{-n} x\right\|^{p_{3}}+\left\|2^{-n} y\right\|^{p_{3}}\right)+\eta_{3}=2^{-p_{3} n} \varepsilon_{3}\left(\|x\|^{p_{3}}+\|y\|^{p_{3}}\right)+\eta_{3}
\end{aligned}
$$

and, consequently,

$$
\left\|2^{2 n} g(0)-g(x) y+g(y) x-x d(y)+y d(x)\right\| \leq 2^{\left(2-p_{3}\right) n} \varepsilon_{3}\left(\|x\|^{p_{3}}+\|y\|^{p_{3}}\right),
$$

whence, with $n \rightarrow \infty$, we get (4.9).

It is easily seen that from (4.2), (4.8), and (4.9) we obtain

$$
G([x, y])=G(x) y-G(y) x+x D(y)-y D(x), \quad x, y \in A .
$$

Now, we can complete the proof analogously as in the proof of Theorem 3.1. Namely, for every $z, w \in \mathcal{A}$, there exist $z_{1}, z_{2}, w_{1}, w_{2} \in A$ with $z=z_{1}-z_{2}$, 
$w=w_{1}-w_{2}$ and, consequently, by (4.10) and by the additivity of $G$ and $D$, we have

$$
\begin{aligned}
G([z, w])= & G\left(\left[z_{1}-z_{2}, w_{1}-w_{2}\right]\right) \\
= & G\left(\left[z_{1}, w_{1}\right]\right)-G\left(\left[z_{1}, w_{2}\right]\right)-G\left(\left[z_{2}, w_{1}\right]\right)+G\left(\left[z_{2}, w_{2}\right]\right) \\
= & G\left(z_{1}\right) w_{1}-G\left(w_{1}\right) z_{1}+z_{1} D\left(w_{1}\right)-w_{1} D\left(z_{1}\right) \\
= & G\left(z_{1}\right) w_{1}-G\left(w_{1}\right) z_{1}+z_{1} D\left(w_{1}\right)-w_{1} D\left(z_{1}\right) \\
& -G\left(z_{1}\right) w_{2}+G\left(w_{2}\right) z_{1}-z_{1} D\left(w_{2}\right)+w_{2} D\left(z_{1}\right) \\
& -G\left(z_{2}\right) w_{1}+G\left(w_{1}\right) z_{2}-z_{2} D\left(w_{1}\right)+w_{1} D\left(z_{2}\right) \\
& +G\left(z_{2}\right) w_{2}-G\left(w_{2}\right) z_{2}+z_{2} D\left(w_{2}\right)-w_{2} D\left(z_{2}\right) \\
= & G\left(z_{1}-z_{2}\right)\left(w_{1}-w_{2}\right)-G\left(w_{1}-w_{2}\right)\left(z_{1}-z_{2}\right) \\
& +\left(z_{1}-z_{2}\right) D\left(w_{1}-w_{2}\right)-\left(w_{1}-w_{2}\right) D\left(z_{1}-z_{2}\right) \\
= & G(z) w-G(w) z+z D(w)-w D(z) .
\end{aligned}
$$

In this way we have shown that $G$ is a generalized Lie $D$-derivation. The proof is completed.

The next two theorems concern some cases that are complementary to those described in (a)-(c). For the sake of simplicity we present them only for $A=\mathcal{A}$ or, when necessary, for $A=\mathcal{A} \backslash\{0\}$. As before, we assume that $g: \widetilde{A}_{0} \rightarrow \mathcal{M}$ and $d: A \rightarrow \mathcal{M}$ are mappings satisfying conditions (3.1)-(3.3).

Theorem 4.2. Let $A=\mathcal{A}$ and $s:=\sup \{|\gamma|: \gamma \in \Gamma\}<\infty$. If there is $\gamma_{0} \in \Gamma$ with $\left|\gamma_{0}\right|=1, \eta_{1}=\eta_{2}=\eta_{3}=0, p_{1}>1, p_{2}>1$, and $p_{3}>2$, then there exist a unique additive mapping $D: \mathcal{A} \rightarrow \mathcal{M}$ and a unique generalized Lie $D$-derivation $G: \mathcal{A} \rightarrow \mathcal{M}$ such that (3.10) holds and

$$
\|d(x)-D(x)\| \leq \frac{\rho_{1} \varepsilon_{1}\|x\|^{p_{1}}}{1-\rho_{1}}, \quad\|g(x)-G(x)\| \leq \frac{\rho_{2} \varepsilon_{2}\|x\|^{p_{2}}}{1-\rho_{2}}
$$

for all $x \in \mathcal{A}$, where

$$
\rho_{i}:=\inf \left\{|c|^{p_{i}} s^{1-p_{i}}+|1-c|^{p_{i}}: c \in \mathbb{F}\right\}, \quad i=1,2 .
$$

Moreover, if $\Gamma$ has a subset from $\mathfrak{B}_{\mathbb{F}}$, then $G$ and $D$ are $\mathbb{F}$-linear.

Proof. Taking $x=y=0$ in (3.1) and (3.2) we get

$$
g(0)=0, \quad d(0)=0 .
$$

Next, since $p_{i}>1$ for $i=1,2, p_{3}>2$, and $s \geq 1$, there are $c \in \mathbb{F} \backslash\{0\}$ and $\mu \in \Gamma \backslash\{0\}$ with

$$
\begin{gathered}
|c|^{p_{i}}|\mu|^{1-p_{i}}+|1-c|^{p_{i}}<1, \quad i=1,2, \\
(|\mu|+1)\left(|c|^{p_{3}}|\mu|^{1-p_{3}}+|1-c|^{p_{3}}\right)<1 .
\end{gathered}
$$

For example, we can choose $c=1 / 2$ and $\mu=\gamma_{0}$. 
It is easy to check that conditions (3.4) and (3.5) hold with the constant function $\xi_{j}$, defined by

$$
\xi_{j}(x, y) \equiv|c|^{p_{j}}|\mu|^{1-p_{j}}+|1-c|^{p_{j}}, \quad j=1,2 .
$$

Further, for every $z, w \in \mathcal{A}$, we have

$$
\begin{array}{r}
\widehat{\Lambda}_{c}^{\mu} \varphi_{3}(z, w)=\varepsilon_{3}(|\mu|+1)\left(|c|^{p_{3}}|\mu|^{1-p_{3}}+|1-c|^{p_{3}}\right)\left(\|z\|^{p_{3}}+\|w\|^{p_{3}}\right) \\
=(|\mu|+1)\left(|c|^{p_{3}}|\mu|^{1-p_{3}}+|1-c|^{p_{3}}\right) \varphi_{3}(z, w),
\end{array}
$$

whence, on account of (4.13),

$$
\lim _{n \rightarrow \infty}\left(\widehat{\Lambda}_{c}^{\mu}\right)^{n} \varphi_{3}(z, w)=0 .
$$

By Theorem 3.1 (i) (with $A=\mathcal{A}$ ) and by (4.12), there exist a unique additive $D: \mathcal{A} \rightarrow \mathcal{M}$ and a unique generalized Lie $D$-derivation $G: \mathcal{A} \rightarrow \mathcal{M}$ fulfilling (3.10) and such that

$$
\|d(x)-D(x)\| \leq \Phi_{1}(x), \quad\|g(x)-G(x)\| \leq \Phi_{2}(x), \quad x \in \mathcal{A},
$$

with

$$
\Phi_{i}(x)=\frac{|c|^{p_{i}}|\mu|^{1-p_{i}}+|1-c|^{p_{i}}}{1-|c|^{p_{i}}|\mu|^{1-p_{i}}-|1-c|^{p_{i}}} \varepsilon_{i}\|x\|^{p_{i}}, \quad x \in \mathcal{A}, i=1,2 .
$$

Moreover, by Theorem 2.6 (i), for every $c_{0} \in \mathbb{F} \backslash\{0\}$ and $\mu_{0} \in \Gamma \backslash\{0\}$ with

$$
\left\|c_{0}\right\|^{p_{i}}\left|\mu_{0}\right|^{1-p_{i}}+\left\|1-c_{0}\right\|^{p_{i}}<1, \quad i=1,2,
$$

there exist unique additive $D_{0}, G_{0}: \mathcal{A} \rightarrow \mathcal{M}$ such that

$$
\begin{gathered}
G_{0}(\gamma x)=\gamma G_{0}(x), \quad D_{0}(\gamma x)=\gamma D_{0}(x), \quad x \in \mathcal{A}, \gamma \in \Gamma, \\
\left\|d(x)-D_{0}(x)\right\| \leq \widehat{\Phi}_{1}(x), \quad\left\|g(x)-G_{0}(x)\right\| \leq \widehat{\Phi}_{2}(x), \quad x \in \mathcal{A},
\end{gathered}
$$

where

$$
\widehat{\Phi}_{i}(x)=\frac{\left\|c_{0}\right\|^{p_{i}}\left|\mu_{0}\right|^{1-p_{i}}+\left\|1-c_{0}\right\|^{p_{i}}}{1-\left\|c_{0}\right\|^{p_{i}}\left|\mu_{0}\right|^{1-p_{i}}-\left\|1-c_{0}\right\|^{p_{i}}} \varepsilon_{i}\|x\|^{p_{i}}, \quad x \in \mathcal{A}, i=1,2 .
$$

Clearly, the uniqueness of $G, G_{0}, D$, and $D_{0}$ means that $G=G_{0}$ and $D=D_{0}$, whence (4.11) is valid. The proof is completed.

TheOrem 4.3. Let $A=\mathcal{A} \backslash\{0\}, \sigma:=\sup \{|\mu+1|: \mu \in \Gamma\}>1, p_{1}<1$, $p_{2}<1, p_{3}<2$, and suppose that $\Gamma$ is bounded. Then there are a unique additive mapping $D: \mathcal{A} \rightarrow \mathcal{M}$ and a unique generalized Lie D-derivation $G: \mathcal{A} \rightarrow \mathcal{M}$ such that (3.10) holds and

$$
\begin{array}{ll}
\|d(x)-D(x)\| \leq \frac{2 \varepsilon_{1}\|x\|^{p_{1}}}{\sigma-\sigma^{p_{1}}}+\frac{\eta_{1}}{\sigma-1}, & x \in \mathcal{A} \backslash\{0\}, \\
\|g(x)-G(x)\| \leq \frac{2 \varepsilon_{2}\|x\|^{p_{2}}}{\sigma-\sigma^{p_{2}}}+\frac{\eta_{2}}{\sigma-1}, & x \in \mathcal{A} \backslash\{0\} .
\end{array}
$$

Moreover, if $\Gamma$ has a subset from $\mathfrak{B}_{\mathbb{F}}$, then $G$ and $D$ are $\mathbb{F}$-linear. 
Proof. Take $\mu \in \Gamma$ with $|\mu+1|>1$. Note that

$$
\begin{gathered}
\bar{\varphi}_{i}(x):=\sum_{n=0}^{\infty} \frac{\varphi_{i}\left((\mu+1)^{n} x,(\mu+1)^{n} x\right)}{|\mu+1|^{n+1}} \\
=\frac{2}{|\mu+1|} \sum_{n=0}^{\infty}|\mu+1|^{n\left(p_{i}-1\right)} \varepsilon_{i} \|\left. x\right|^{p_{i}}+\sum_{n=0}^{\infty} \frac{\eta_{i}}{|\mu+1|^{n+1}} \\
=\frac{2 \varepsilon_{i} \|\left. x\right|^{p_{i}}}{|\mu+1|-|\mu+1|^{p_{i}}}+\frac{\eta_{i}}{|\mu+1|-1}, \quad x \in A, \\
\lim _{n \rightarrow \infty} \frac{\varphi_{i}\left((\mu+1)^{n} x,(\mu+1)^{n} y\right)}{|\mu+1|^{n}}, \quad x, y \in A, i=1,2, \\
=\lim _{n \rightarrow \infty} \frac{\left\|\left.x\right|^{p_{i}}+\right\| y \|^{p_{i}}}{|\mu+1|^{n\left(1-p_{i}\right)}}+\frac{\eta_{i}}{|\mu+1|^{n}}=0, \\
\lim _{n \rightarrow \infty} \frac{\varphi_{3}\left((\mu+1)^{n} z,(\mu+1)^{n} w\right)}{|\mu+1|^{2 n}} \\
=\lim _{n \rightarrow \infty} \varepsilon_{3} \frac{\|z\|^{p_{3}}+\|\left. w\right|^{p_{3}}}{|\mu+1|^{n\left(2-p_{3}\right)}}+\frac{\eta_{3}}{|\mu+1|^{2 n}}=0, \quad z, w \in A .
\end{gathered}
$$

Hence, by Theorem 3.3, there exist a unique additive $D_{\mu}: \mathcal{A} \rightarrow \mathcal{M}$ and a unique generalized Lie $D_{\mu}$-derivation $G_{\mu}: \mathcal{A} \rightarrow \mathcal{M}$ such that (3.10) and (3.22) hold with $D$ and $G$ replaced by $D_{\mu}$ and $G_{\mu}$, respectively. Moreover, if $\Gamma$ has a bounded subset belonging to $\mathfrak{B}_{\mathbb{F}}$, then $D_{\mu}$ and $G_{\mu}$ are $\mathbb{F}$-linear.

Clearly, the uniqueness of $D_{\mu}$ and $G_{\mu}$ implies that $D:=D_{\mu}=D_{\nu}$ and $G:=G_{\mu}=G_{\nu}$ for all $\nu \in \Gamma$ with $|\nu+1|>1$. Thus, (4.16) and (4.17) are valid and the proof is completed.

\section{REFERENCES}

[1] M. Amyari, C. Baak and M. S. Moslehian, Nearly ternary derivations, Taiwanese J. Math. 11 (2007), 1417-1424.

[2] T. Aoki, On the stability of the linear transformation in Banach spaces, J. Math. Soc. Japan 2 (1950), 64-66.

[3] R. Badora, On approximate derivations, Math. Inequal. Appl. 9 (2006), 167-173.

[4] Z. Boros and E. Gselmann, Hyers-Ulam stability of derivations and linear functions, Aequationes Math. 80 (2010), 13-25.

[5] N. Brillouët-Belluot, J. Brzdęk and K. Ciepliński, On some recent developments in Ulam's type stability, Abstr. Appl. Anal. 2012 (2012), Article ID 716936, 41 pages.

[6] J. Brzdęk, Remarks on hyperstability of the Cauchy functional equation, Aequationes Math., DOI 10.1007/s00010-012-0168-4.

[7] J. Brzdęk, J. Chudziak and Z. Páles, A fixed point approach to stability of functional equations, Nonlinear Anal. 74 (2011), 6728-6732.

[8] J. Brzdęk and A. Fošner, Remarks on the stability of Lie homomorphisms, J. Math. Anal. Appl. 400 (2013), 585-596.

[9] P. Fischer and Z. Słodkowski, Christensen zero sets and measurable convex functions, Proc. Amer. Math. Soc. 79 (1980), 449-453. 
[10] M. E. Gordji and M. S. Moslehian, A trick for investigation of approximate derivations, Math. Commun. 15 (2010), 99-105.

[11] S. Hejazian, H. Mahdavian Rad and M. Mirzavaziri, $(\delta, \epsilon)$-double derivations on Banach algebras, Ann. Funct. Anal. 1 (2010), 103-111.

[12] B. Hvala, Generalized Lie derivations on prime rings, Taiwan. J. Math 11 (2007), $1425-1430$

[13] D. H. Hyers, On the stability of the linear functional equation, Proc. Natl. Acad. Sci. U.S.A. 27 (1941), 222-224.

[14] D. H. Hyers, G. Isac and Th. M. Rassias, Stability of functional equations in several variables, Birkhäuser, Boston, 1998.

[15] W. Jabłoński, On a class of sets connected with a convex function, Abh. Math. Sem. Univ. Hamburg 69 (1999), 205-210.

[16] W. Jabłoński, Sum of graphs of continuous functions and boundedness of additive operators, J. Math. Anal. Appl. 312 (2005), 527-534.

[17] S.-M. Jung, Hyers-Ulam-Rassias stability of functional equations in nonlinear analysis, Springer Optimization and Its Applications, 48. Springer, New York, 2011.

[18] K.-W. Jun and D.-W. Park, Almost derivations on the Banach algebra $C^{n}[0,1]$, Bull. Korean Math. Soc. 33 (1996), 359-366.

[19] M. Kuczma, An Introduction to the Theory of Functional Equations and Inequalities: Cauchys Equation and Jensens Inequality (Second Edition), Birkhäuser, Boston, 2009.

[20] M. Mirzavaziri and M. S. Moslehian, Automatic continuity of $\sigma$-derivations on $C^{*}$ algebras, Proc. Amer. Math. Soc. 134 (2006), 3319-3327.

[21] T. Miura, H. Oka, G. Hirasawa and S.-E. Takahasi, Superstability of multipliers and ring derivations on Banach algebras, Banach J. Math. Anal. 1 (2007), 125-130.

[22] M. S. Moslehian, Ternary derivations, stability and physical aspects, Acta Appl. Math. 100 (2008), 187-199.

[23] A. Nakajima, On generalized higher derivations, Turkish J. Math. 24 (2000), 295-311.

[24] C. Park, Linear derivations on Banach algebras, Nonlinear Funct. Anal. Appl. 9 (2004), 359-368.

[25] Th. M. Rassias, On the stability of the linear mappings in Banach spaces, Proc. Amer. Math. Soc. 72 (1978), 297-300.

[26] S. M. Ulam, Problems in modern mathematics, John Wiley \& Sons, New York, 1964.

\author{
J. Brzdęk \\ Department of Mathematics \\ Pedagogical University \\ Podchorążych 2, 30-084 Kraków \\ Poland \\ E-mail: jbrzdek@up.krakow.pl
}

A. Fošner

Faculty of Management

University of Primorska

Cankarjeva 5, SI-6104 Koper

Slovenia

E-mail: ajda.fosner@fm-kp.si

Received: 8.6.2014.

Revised: 14.7.2014. 